Ann. Zootech., I972, 21 (4), 55I-566.

\title{
ALIMENTATION DES VACHES LAITIÈRES AVEC DES RATIONS A PROPORTION VARIABLE D'ALIMENTS CONCENTRÉS : PRODUCTION ET COMPOSITION DU LAIT, ET DIGESTION DANS LE RUMEN
}

\author{
B. REMMOND \\ avec la collaboration technique de Jeanne Fléchet, A. Ollier et Louise Toullec \\ Station de Recherches sur l'Élevage des Ruminants \\ Centre de Recherches de Clermont-Ferrand, I. N. R. A., \\ Saint Genès Champanelle \\ 63110 Beaumont
}

RÉSUMÉ

Dix vaches normales et deux vaches porteuses d'une large fistule du rumen, toutes en lactation, ont reçu au cours de 5 ou 6 périodes expérimentales successives des régimes distribués en quantités fixées dont la proportion d'aliment concentré est passée de 50 ou 40 p. Ioo à $90 \mathrm{p}$. Ioo, par augmentation de ro p. Ioo à chaque période. Après la dernière période expérimentale, les animaux ont été ramenés en 2 jours à un régime comportant 50 p. roo de foin dans la ration (période post-expérimentale).

Les animaux ont toujours ingéré presque toute la ration qui leur était offerte dans les 2 heures qui suivaient la distribution des aliments (tabl. 2 et 4 ). La quantité de lait à 4 p. Ioo de matières grasses produite a diminué légèrement plus rapidement que l'équivalent en lait de l'apport d'énergie nette $(0,48 \mathrm{~kg}$ contre $0,44 \mathrm{~kg}$ par semaine, fig. I). Le taux butyreux a diminué de façon linéaire (de $\mathrm{r}, 5 \mathrm{~g} \mathrm{p}$. I ooo par période), mais il est redevenu presque normal au cours de la $\mathbf{2}^{\mathrm{e}}$ semaine de la période post-expérimentale. Le poids vif a suivi une évolution semblable à celle du taux butyreux. Le bilan énergétique des animaux a toujours été positif, de r, I UF en moyenne au cours des différentes périodes expérimentales et la teneur du plasma en acides gras libres a été plutôt faible (tabl. 3). La proportion de l'acide acétique dans le mélange des acides gras volatils a diminué quand la proportion de l'aliment concentré dans la ration a augmenté, et celle de l'acide propionique a augmenté, mais ces modifications ont été relativement limitées (tabl. 6).

D'une façon générale, les modifications de la digestion dans le rumen et de la production et de la composition du lait ont été beaucoup plus faibles que celles observées au cours des 2 essais précédents (RÉmond et JoURNET, I97 I et 1972). 


\section{INTRODUCTION}

Dans deux essais précédents (RÉMOND et JOURNET, I97 I et I972), 1'alimentation de vaches laitières avec du foin et un aliment concentré tous deux distribués à volonté avait provoqué une diminution rapide de la quantité de lait produite et du taux butyreux du lait, et un engraissement important des animaux; il en était résulté une faible efficacité de l'énergie pour la production laitière. Nous nous sommes demandés si ces modifications étaient dues au pourcentage élevé de l'aliment concentré dans la ration (environ 85 p. Ioo) ou au fait que les vaches recevaient cet aliment à volonté et en ingéraient des quantités élevées.

L'efficacité d'utilisation des aliments pour la production de lait est généralement exprimée par le rapport de la quantité d'énergie ingérée (totale ou d.sponible pour la production) à la quantité de lait de référence produite, ou par le rapport inverse. Dans le système de l'énergie digestible (BLOOM et al., I955 ; ELLio'T'T et LoOSLI, I959) ou métabolisable (Coppock et al., I 964), la distribution en quantité limitée de rations à proportion croissante d'aliment concentré a entraîné une augmentation de l'efficacité d'utilisation de l'énergie sauf dans un essai réalisé par Swanson, Hinton et MrLes (I967) pendant toute une lactation. Au contraire, la distribution à volonté d'aliment concentré (comparée à une distribution selon des recommandations) ou de rations broyées et agglomérées dans lesquelles la proportion d'aliment concentré augmentait, a provoqué une diminution de l'efficacité de l'utilisation de l'énergie pour la production de lait (PUTNam et LoOshI, I959; Ronning, I96o ; Brown et al., I962 ; Bishop et al., I963; ETGEN et al., I965; Or,SON, HiNnERs et BERNETT, I966) ; cependant, BELL, Horton et STALLCUP (I963) n'ont pas observé de différence. Dans le système de l'énergie nette, l'efficacité de l'utilisation de l'énergie de la ration pour la production laitière n'a, en général, pas varié avec la proportion d'aliment concentré quand les quantités de ration ou d'aliment concentré étaient distribuées en quantité limitée (MARTIN, STODdARD et ALLEN, I954; Bloom et al., I955 ; FiLLIOTT et L,OOSLI, I959; Hinders et OWEn, I963 ; Zeremski et al., I965) ; cependant Morris, GaINER et DAvis (I958) ont observé une diminution d'efficacité. Au contraire, quand la ration ou l'aliment concentré étaient offerts à volonté, l'efficacité d'utilisation de l'énergie nette a diminué (Ronning, I960; Hooven et Plowman, I963).

L'effet sur le taux butyreux du niveau de distribution de régimes de composition déterminée a été peu étudié. NORDFELDT et RUUDVERE (I963) n'ont pas observé de modification du taux butyreux quand ils distribuaient à 3 niveaux $(0,3,0,4$ et 0,5 UF par $\mathrm{kg}$ de lait à $4 \mathrm{p}$. Ioo de matières grasses) 4 rations dont les rapports : UF apportées par le fourrage/UF apportées par 1'aliment concentré étaient $70 / 30$, $55 / 45,40 / 60$ et $25 / 75$.

I1 semble done que l'augmentation de la proportion de l'aliment concentré dans une ration distribuée en quantité limitée modifie peu l'efficacité alimentaire de la ration (dans le système de l'énergie nette) et modifie moins la composition du lait et probablement les phénomènes de la digestion dans le rumen que dans le cas d'une distribution ad libitum. C'est ce que nous avons voulu vérifier dans cet essai. 


\section{MATÉRIEL ET MÉTHODES}

Nous avons réalisé 2 essais : un essai d'alimentation avec Io vaches normales et un essai de digestion avec 2 vaches porteuses d'une large fistule du rumen.

\section{Essai d'alimentation}

\section{Dispositif expérimental et alimentation.}

Les Io vaches utilisées (9 Holstein et I Pie Rouge) étaient en moyenne à leur $20^{e}$ jour de lactation au début de l'essai qui s'est déroulé entre le $\mathrm{I} 2$ février et le 7 juillet $1968 ; 8$ d'entre elles étaient en première lactation et deux en seconde.

Après une période pré-expérimentale de 4 semaines dont la dernière était une semaine de transition, les animaux ont reçu au cours de 5 périodes expérimentales successives de 3 semaines chacune (appelées période I, 2, 3, 4 et 5 dans la suite de l'exposé) des régimes dont le pourcentage d'aliment concentré dans la matière sèche est passé de 50 à $90 \mathrm{p}$. roo, par une augmentation de Io $\mathrm{p}$. Ioo d'une période à la suivante (ils sont appelés régimes $\mathrm{C} 5 \mathrm{o}, \mathrm{C} 60, \mathrm{C} 7 \mathrm{o}, \mathrm{C} 80$ et $\mathrm{C} 90$ dans la suite de l'exposé). Après la dernière période expérimentale, les animaux ont été ramenés en 2 jours, au début de la semaine de transition à un régime contenant 50 p. Io d'aliment concentré et y ont été maintenus au cours des 2 semaines suivantes qui ont constitué la période post-expérimentale.

Pendant la période pré-expérimentale, les animaux recevaient une ration composée de foin, d'ensilage de maiss et d'aliment concentré dont la quantité distribuée était ajustée à leurs besoins. Pendant les périodes expérimentales et la période post-expérimentale ils ont reçu des quantités fixées à l'avance d'un aliment concentré et de 2 foins de telle sorte que le rapport quantité de foin/quantité d'aliment concentré fût celui qu'on s'était fixé, et que les apports énergétiques couvrissent environ I I5 p. Ioo des besoins théoriques d'entreticn et de production laitière des animaux.

Les besoins théoriques des animaux ont été calculés à partir : $\mathrm{I}^{0}$ ) de leur poids vif à la fin de la période pré-expérimentale, en utilisant la recommandation suivante : UF pour l'entretien $=\mathbf{I}, 5+$ poids vif en centaines de $\mathrm{kg} / 2$, et $2^{\circ}$ ) de leur production laitière théorique (calculée en supposant une diminution de la quantité de lait produite de $0,4 \mathrm{~kg}$ par semaine si elle était supérieure à $20 \mathrm{~kg}$ et de $0,3 \mathrm{~kg}$ dans le cas contraire), en utilisant la recommandation suivante : 0,38 UF par $\mathrm{kg}$ de lait à $4 \mathrm{p}$. Ioo de matières grasses.

Les vaches étaient en permanence en stabulation entravée sur une litière de copeaux de bois et recevaient un foin et la moitié de l'aliment concentré le matin à $7 \mathrm{~h} 45$, l'autre foin et l'autre moitié de l'aliment concentré l'après-midi à 15 h 15 . Les refus étaient enlevés 2 fois par jour.

\section{TABLEAU I}

Composition chimique et valeur énergétique des aliments

\begin{tabular}{|c|c|c|c|c|}
\hline \multirow[b]{2}{*}{ Aliments } & \multicolumn{3}{|c|}{$\begin{array}{c}\text { Composition chimique } \\
\text { (p. } 100 \text { de la matière sèche) }\end{array}$} & \multirow{2}{*}{$\begin{array}{l}\text { Valeur énergétique } \\
\text { (UF/kg de } \\
\text { matière sèche) }\end{array}$} \\
\hline & $\begin{array}{l}\text { Matière } \\
\text { organique }\end{array}$ & $\begin{array}{c}\text { Matières } \\
\text { azotées totales } \\
(\mathrm{N} \times 6,25)\end{array}$ & $\begin{array}{l}\text { Cellulose } \\
\text { brute }\end{array}$ & \\
\hline Foin de ray-grass ( $2^{e}$ coupe) $\ldots$ & 90,4 & 11,6 & 33,4 & 0,59 \\
\hline Foin de ray-grass (jeune semis) & 88,3 & 12,3 & 29,4 & 0,68 \\
\hline Aliment concentré $\ldots \ldots \ldots \ldots$ & 91,7 & 18,9 & 7,9 & 0,97 \\
\hline
\end{tabular}


Les 2 foins de ray-grass, l'un de jeune semis, l'autre de $2^{\mathrm{e}}$ coupe, étaient distribués sous forme normale et l'aliment concentré sous forme broyée; ce dernier avait la composition suivante : orge $=50$ p. IOO, avoine $=22$ p. IOO, tourteau de colza $=$ I 5 p. IOO, tourtcau d'arachide $=8$ p. IOO, composé minéral $(65 \mathrm{p}$. Ioo de phosphate bicalcique et $35 \mathrm{p}$. Ioo de sel marin) $=5 \mathrm{p}$. Ioo. La composition chimique des aliments et leur valeur énergétique sont données dans le tableau $\mathbf{I}$. La valeur énergétique des foins a été estimée par la formule de BRErREM (I954) à partir de la digestibilité de leur matière organique, mesurée avec 4 moutons ; celle de l'aliment concentré a été calculée à partir de la valeur énergétique de chacun de ses constituants donnée par les tables de Leroy.

\section{Mesures.}

Les quantités d'aliments ingérées (exprimées en poids de matière sèche) ont été obtenues par pesée des aliments distribués et refusés et détermination deux fois par semaine de leur teneur en matière sèche. La quantité de lait produite a été peséc à chaque traite. Le taux butyreux du lait a été mesuré chaque jour par la méthode GERBER sur un échantillon moyen du lait des 2 traites, et sa teneur en protéines une fois par semaine par la méthode au Noir Amido sur un échantillon moyen du lait de 4 traites consécutives. Les animaux ont été pesés une fois par semaine.

Nous avons mesuré la teneur du sang en corps cétoniques (Procos, r96r) et celle du plasma en acides gras libres (Dole et Mernertz, ig60) et en glucose (HugGetT et Nixon, 1957), sur 6 des Io vaches. Les prises de sang ont été effectuées au niveau de la veine jugulaire, 3 heures après la distribution des aliments du matin. Elles ont eu lieu pendant la période expérimentale, une fois au cours de chaque période, et pendant la période post-expérimentale, 2 fois au cours de la semaine de transition et une fois au cours des 2 semaines suivantes.

\section{Essais de digestion}

\section{Dispositif expérimental et alimentation.}

Les 2 vaches fistulées utilisées (3I65 et 55 I 6 ) étaient de race $F . F . P . N$. et toutes deux à leur $69^{\mathrm{e}}$ jour de lactation au début de l'essai qui s'est déroulé entre le 22 janvier et le 9 juin I968; elles ont reçu au cours de 6 périodes expérimentales successives de 3 semaines chacune des régimes dont le pourcentage d'aliment concentré est passé de 40 à go p. Ioo par augmentation de ro p. Ioo d'une période à la suivante. Elles ont été alimentées à roo p. Ioo de leurs besoins théoriques (calculés de la même façon que dans l'essai d'alimentation) jusqu'au milieu de la période 3 (6o C) et i 5 p. roo ultérieurement. Ce rajustement a été effectué au vu de la perte de poids vif des animaux pendant les 2 premières périodes expérimentales.

Les aliments étaient les mêmes que ceux de l'essai d'alimentation.

Les 2 vaches étaient en permanence en stabulation entravée sur des stalles revêtues de plaques en caoutchouc. Contrairement aux vaches normales, les vaches fistulées recevaient le foin une fois que l'aliment concentré avait été presque totalement ingéré. Ce dernier était distribué le matin à $8 \mathrm{~h} 30$ et le soir à $18 \mathrm{~h} 30$.

\section{Mesures.}

Les quantités ingérées, les quantités de lait produites, la composition du lait, et le poids vif ont été mesurés de la même façon et avec la même fréquence que pour les vaches normales.

Les prélèvements de sang destinés à la détermination des teneurs en corps cétoniques et en acides gras libres ont eu lieu avant la distribution des aliments du matin et 3 heures après, 2 jours consécutifs au cours de la dernière semaine de chaque période expérimentale et 2 jours au cours de chacune des 3 semaines de la période post-expérimentale.

De plus, on a mesuré le $\mathrm{pH}$ du jus de rumen avant la distribution des aliments du matin et $3 \mathrm{~h}, 7 \mathrm{~h}$ et Io $\mathrm{h}$ après. Sur le jus de rumen prélevé avant la distribution des aliments et 3 heures après on a aussi mesuré la concentration et la composition du mélange des acides gras volatils (AGV), par la méthode de RrGaud et Journet (I970). Ces prélèvements avaient lieu 3 jours consécutifs pendant la dernière semaine de chaque période expérimentale, et pendant la période post-expérimentale chaque jour de la semaine de transition et 2 jours au cours de chacune des 2 semaines suivantes. L'emploi du temps des animaux a été enregistré pendant au moins 4 jours consécutifs au cours de chaque période expérimentale par la méthode de Ruckebusch (I963). 


\section{RÉSULTA'TS}

\section{Essai d'alimentation}

Quantités d'aliments ingérées.

Les quantités de foin et d'aliment concentré ingérées ont été presque égales aux quantités distribuées, les quantités refusées ayant toujours été faibles (tab1. 2). Entre les périodes I et 5 , les quantités de foin ingérées sont passées de $7,17 \mathrm{~kg}$ à $\mathrm{I}, 22 \mathrm{~kg}$ et les quantités d'aliment concentré de $7,04 \mathrm{~kg}$ à $9,27 \mathrm{~kg}$. Les aliments refusés étaient essentiellement constitués de foin avec les régimes $\mathrm{C} 50$ et $\mathrm{C} 60$ et d'aliment concentré avec les régimes $\mathrm{C} 70, \mathrm{C} 80$ et $\mathrm{C} 90$.

TABLEAU 2

Quantités journalières d'aliments ingérées et refusées Essai d'alimentation

\begin{tabular}{|c|c|c|c|c|c|c|c|c|}
\hline \multirow{2}{*}{ Période } & \multirow{2}{*}{$\begin{array}{l}\text { Pré-expéri- } \\
\text { mentale }\end{array}$} & \multicolumn{5}{|c|}{ Expérimentale } & \multirow{2}{*}{\multicolumn{2}{|c|}{$\begin{array}{l}\text { Post-expéri- } \\
\text { mentale }\end{array}$}} \\
\hline & & 1 & 2 & 3 & $l_{\mathbf{t}}$ & 5 & & \\
\hline $\begin{array}{l}\text { Aliment concentré } \\
\text { (p. } 100 \text { de la ration) }\end{array}$ & & 50 & 60 & 70 & 80 & 90 & 60 & 50 \\
\hline \multicolumn{9}{|l|}{$\begin{array}{c}\text { Quantités ingérées } \\
\text { (kg MS) }\end{array}$} \\
\hline Foin $\ldots \ldots \ldots \ldots \ldots$ & $8,62\left({ }^{1}\right)$ & 7,16 & 5,69 & 3,81 & 2,34 & 1,22 & 4,93 & 6,31 \\
\hline Aliment concentré $\ldots . \ldots$ & 5,38 & 7,04 & 8,37 & 8,93 & 9,20 & 8,94 & 7,10 & 6,25 \\
\hline Total .............. & 14,00 & 14,20 & 14,06 & 12,74 & 11,54 & 10,16 & 12,03 & 12,56 \\
\hline \multicolumn{9}{|l|}{ Aliments refusés } \\
\hline $\begin{array}{c}\text { Aliments refusés : Total } \\
\text { (kg MS) } \ldots \ldots \ldots \ldots \ldots\end{array}$ & & 0,55 & 0,15 & 0,10 & 0,42 & 0,67 & 0,25 & 0,10 \\
\hline $\begin{array}{c}\text { Aliment concentré (p. } 100 \\
\text { des refus) } \ldots \ldots \ldots \ldots\end{array}$ & & 4 & 15 & 100 & 100 & 100 & 20 & 10 \\
\hline
\end{tabular}

(1) Les $8,62 \mathrm{~kg}$ étaient en fait constitués pour moitié de foin pour moitié d'ensilage de maïs.

La quantité d'énergie nette ingérée a diminué en moyenne de o,I7 UF par semaine entre la première semaine expérimentale et la dernière alors que l'objectif était une diminution moyenne de $0, I_{3} \mathrm{UF}$.

$A u$ fur et à mesure que le pourcentage de foin dans la ration diminuait, les vaches ingéraient plus de copeaux, ce qui peut être dû à la diminution de la quantité totale d'aliment ingérée (de $4,04 \mathrm{~kg}$ entre les périodes I et 5 ) et ou à la diminution de fibrosité de la ration. 
Production et composition du lait.

La persistance de la production laitière a été en moyenne de go p. Ioo entre les périodes I et 5, mais a été plus élevée pendant les périodes I et 2 que pendant les périodes 3,4 et 5 ; le taux butyreux a diminué de façon linéaire : d'environ I, $5 \mathrm{~g}$ p. I ooo pour une augmentation de la proportion de l'aliment concentré dans la ration de Io p. Ioo (fig. I). Pendant la période post-expérimentale, la quantité de lait produite a augmenté de plus de $\mathrm{I} \mathrm{kg}$ à cause de l'augmentation de l'apport énergétique aux animaux; le taux butyreux n'a pas varié pendant la semaine de transition par rapport à ce qu'il était avec le régime $\mathrm{C} 90(28,7 \mathrm{~g} \mathrm{p}$. I ooo). Il est revenu à un niveau presque normal (33, I g p. I ooo) au cours de la semaine suivante; il était cependant encore inférieur à celui enregistré pendant la période $I$.

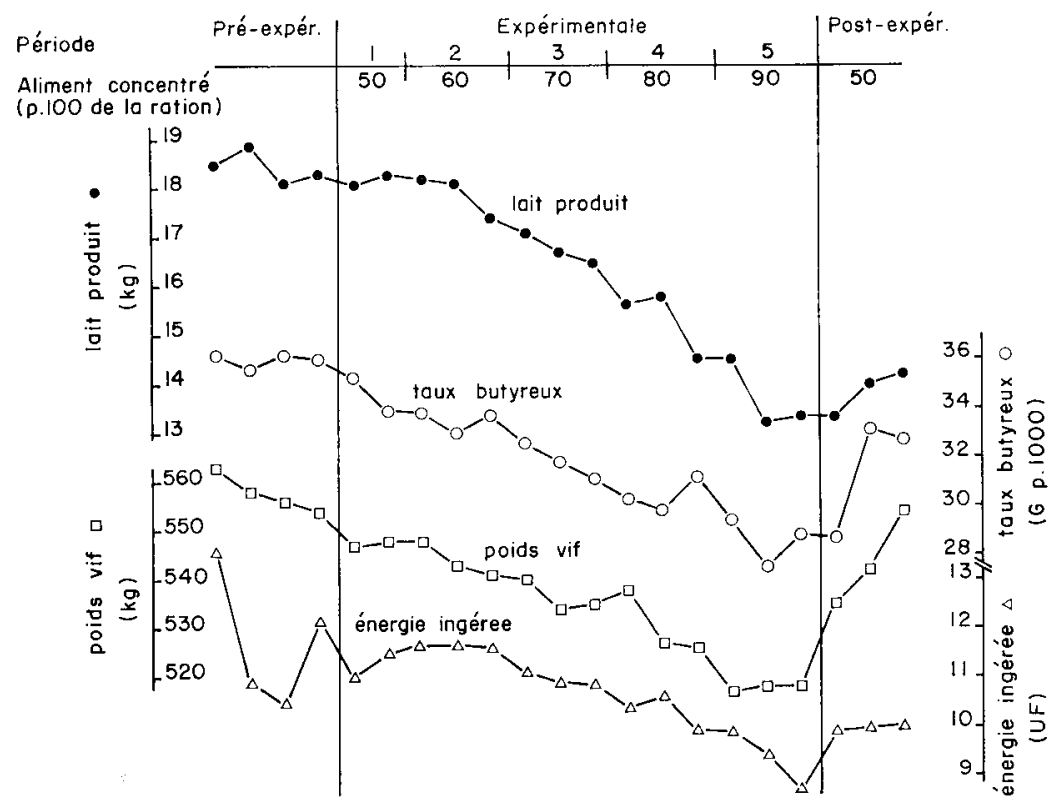

FIG. I. - Evolution de la quantité de lait produite, du taux butyreux, du poids vif des animaux et de la quantité d'énergie ingévée Essai d'alimentation

La teneur en protéines du lait a légèrement augmenté ( $0,9 \mathrm{~g}$ p. I 0oo) entre les régimes $\mathrm{C}_{50}$ et $\mathrm{C} 90$, et a diminué de la même valeur en revenant sur le régime $\mathrm{C}_{50}$ pendant la période post-expérimentale.

$\mathrm{I}_{\mathrm{L}}$ quantité de lait à $4 \mathrm{p}$. Ioo de matières grasses produite a diminué en moyenne de $0,48 \mathrm{~kg}$ par semaine entre les périodes $\mathrm{I}$ et 5 , ce qui correspond à un coefficient de persistance de $87,2 \mathrm{p}$. Ioo par mois. Cette importante diminution est presque complètement expliquée par la diminution hebdomadaire de la quantité d'énergie ingérée, équivalente à $0,44 \mathrm{~kg}$ de lait. La légère différence pourrait provenir du fait que la quantité d'énergie ingérée par les animaux n'a pas diminué régulièrement, mais a augmenté entre les périodes I et 2 , avant de diminuer de façon accélérée jusqu'à la période 5 . 
Poids vif.

Le poids vif des animaux a régulièrement diminué pendant les périodes expérimentales, en moyenne de $2,6 \mathrm{~kg}$ par semaine (fig. I). Puis, entre la dernière semaine de la période 5 et la troisième semaine de la période post-expérimentale (soit en 3 semaines) il a augmenté de $34 \mathrm{~kg}$ et a atteint une valeur supérieure de $7 \mathrm{~kg}$ à celle de la période I alors que la proportion de l'aliment concentré était la même et que les quantités ingérées étaient inférieures de $I, 3 \mathrm{~kg}$. Il est donc vraisemblable que la diminution du poids vif au cours des périodes expérimentales était essentiellement due à une diminution du poids des contenus digestifs.

\section{Bilan énergétique et efficacité des rations.}

On a calculé le bilan énergétique des animaux en estimant d'une part les apports énergétiques et d'autre part les besoins des animaux à partir de la quantité de lait à 4 p. Ioo de matières grasses produite et de leur poids vif ; on a adopté des besoins d'entretien constants, considérant que la variation de poids vif était due à tune variation du poids des contenus digestifs. Excepté pendant une semaine de la période préexpérimentale, le bilan énergétique a toujours été positif de I,I UF en moyenne.

Par UF ingérée au-dessus des besoins d'entretien, les vaches ont produit 2,36 ; $2, \mathrm{I} 5 ; 2, \mathrm{I} 6 ; 2, \mathrm{I} 3 ; 2, \mathrm{I} 9$ et $2, \mathrm{I} 5 \mathrm{~kg}$ de lait à $4 \mathrm{p}$. I00 de matières grasses quand elles recevaient les régimes $50 \mathrm{C}, 60 \mathrm{C}, 70 \mathrm{C}, 80 \mathrm{C}, 90 \mathrm{C}$ et le régime post-expérimental, respectivement. La valeur plus élevée enregistrée avec le régime $50 \mathrm{C}$ pourrait s'expliquer par la diminution importante $(\mathrm{I}, \mathrm{I} 6 \mathrm{UF})$ de la quantité d'énergie ingérée

\section{TABLEAU 3}

Teneurs du sang en corps cétoniques et au plasma en glucose et en acides gras libres Essai d'alimentation

\begin{tabular}{|c|c|c|c|c|c|c|c|c|c|}
\hline \multirow{2}{*}{ Période } & \multicolumn{5}{|c|}{ Expérimentale } & \multicolumn{4}{|c|}{ Post-expérimentale } \\
\hline & 1 & 2 & 3 & 4 & 5 & $\mathrm{~S}_{1}^{*}$ & $\mathrm{~S}_{1}^{*}$ & $\mathrm{~S}_{2} *$ & $\mathrm{~S}_{3}{ }^{*}$ \\
\hline Aliment concentré (p. 100 de la ration) & 50 & 60 & 70 & 80 & 90 & & & 50 & 50 \\
\hline \multicolumn{10}{|l|}{ Glucose (mg/litre de plasma) : } \\
\hline $\begin{array}{l}\text { moyenne } \ldots \ldots \ldots \ldots \ldots \ldots \ldots \\
\text { écart-type } \ldots \ldots \ldots \ldots \ldots \ldots \ldots\end{array}$ & $\begin{array}{r}552 \\
63\end{array}$ & $\begin{array}{r}526 \\
66\end{array}$ & $\begin{array}{r}547 \\
53\end{array}$ & $\begin{array}{r}584 \\
45\end{array}$ & $\begin{array}{r}552 \\
38\end{array}$ & $\begin{array}{r}573 \\
59\end{array}$ & $\begin{array}{r}645 \\
41\end{array}$ & $\begin{array}{r}531 \\
37\end{array}$ & $\begin{array}{r}503 \\
64\end{array}$ \\
\hline \multicolumn{10}{|l|}{ Acides gras libres ( $\mu$-éq/l plasma) : } \\
\hline 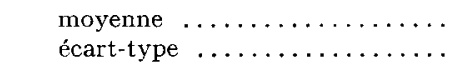 & $\begin{array}{r}241 \\
39\end{array}$ & $\begin{array}{r}213 \\
49\end{array}$ & $\begin{array}{r}216 \\
52\end{array}$ & $\begin{array}{r}223 \\
65\end{array}$ & $\begin{array}{r}201 \\
5^{\prime} t\end{array}$ & $\begin{array}{r}188 \\
36\end{array}$ & $\begin{array}{r}177 \\
18\end{array}$ & $\begin{array}{r}261 \\
28\end{array}$ & $\begin{array}{r}207 \\
25\end{array}$ \\
\hline \multicolumn{10}{|l|}{$\begin{array}{l}\text { Corps cétoniques totaux (mg acétone/ } \\
100 \mathrm{ml} \text { de sang): }\end{array}$} \\
\hline $\begin{array}{l}\text { moyenne } \ldots \\
\text { écart-type .. }\end{array}$ & $\begin{array}{l}2,68 \\
0,53\end{array}$ & $\begin{array}{l}3,83 \\
1,57\end{array}$ & $\begin{array}{l}3,24 \\
1,05\end{array}$ & $\begin{array}{l}2,61 \\
0,59\end{array}$ & $\begin{array}{l}2,10 \\
0,87\end{array}$ & $\begin{array}{l}1,95 \\
0,10\end{array}$ & $\begin{array}{l}2,82 \\
0,77\end{array}$ & $\begin{array}{l}3,22 \\
0,69\end{array}$ & $\begin{array}{l}3,09 \\
0,47\end{array}$ \\
\hline
\end{tabular}

* $\mathrm{S}_{1}, \mathrm{~S}_{2}, \mathrm{~S}_{3}$ : semaines $1,2,3$. 
entre la semaine de transition pré-expérimentale et la première semaine de distribution du régime $50 \mathrm{C}$. Cependant, l'ensemble des valeurs a été relativement faible puisque la valeur théorique est de $2,6 \mathrm{~kg}$ de lait à $4 \mathrm{p}$. Ioo de matières grasses.

\section{Teneur $d u$ sang en certains constituants.}

$\mathrm{L}_{\mathrm{L}} \mathrm{a}$ teneur du plasma en glucose a été en moyenne de $55^{2} \mathrm{mg} / 1$ pendant les périodes expérimentales, et n'a pas varié de façon significative d'un régime à l'autre (tabl. 3). Elle a augmenté pendant la semaine de transition et diminué ensuite. La teneur du plasma en acides gras libres a été relativement faible en moyenne (2I9 $\mu$-éq./1) pendant les périodes expérimentales et n'a pas varié. La teneur du sang en corps cétoniques totaux a augmenté entre les régimes $C_{50}$ et $C 60$, puis a diminué jusqu'à la semaine de transition; elle a remonté pendant la semaine suivante.

\section{Essai de digestion}

Setule la vache $3 \mathrm{I} 65$ a reçu tous les régimes (de $\mathrm{C} 40$ à $\mathrm{C}$ 9o). La vache $55 \mathrm{I} 6$ a dû être retirée d'expérience pour troubles digestifs alors qu'elle recevait le régime $\mathrm{C} 80$.

\section{Comportement alimentaire.}

Les périodes d'ingestion ont toujours été groupées après chacune des deux distributions d'aliments, en particulier chez la vache $3 \mathrm{I} 65$ qui, sauf avec le régime $\mathrm{C}_{5} \mathrm{O}$, a toujours ingéré sa ration dans les 2 heures qui suivaient la distribution des aliments.

Les durées journalières d'ingestion et de rumination (exprimées en p. Ioo du temps total) ont commencé par diminuer quand le pourcentage d'aliment concentré dans la ration a augmenté. Mais cette évolution, et principalement celle qui concerne la durée de rumination (vache $3 \mathrm{r} 65$ ), s'est arrêtée et peut-être même inversée pour les régimes comportant un pourcentage très élevé d'aliment concentré (tabl. 4).

Les durées unitaires d'ingestion et de rumination (durée d'ingestion et de rumination par $\mathrm{kg}$ de matière sèche ingérée) ont varié dans le même sens que les durées journalières d'ingestion et de rumination. Pendant la période post-expérimentale, la durée unitaire d'ingestion a été beaucoup plus courte que pendant la première période expérimentale alors que les régimes avaient la même composition.

La comparaison des résultats de cet essai avec ceux de l'essai précédent (RÉMOND et JOURNET, I972) dans lequel les animaux recevaient les aliments à volonté, indique que la limitation des quantités d'aliments distribués a entrainé une diminution du nombre de périodes d'ingestion, qui est passé de 15 à 2 environ, une réduction de moitié de la durée unitaire d'ingestion qui est passée de 23 à I2 minutes par $\mathrm{kg}$ de matière sèche totale ingérée et une réduction plus faible de la durée unitaire de rumination.

\section{Production laitière.}

La diminution de la quantité de lait produite $(3,7 \mathrm{~kg})$ et du taux butyreux $(8,7 \mathrm{~g} \mathrm{p}$. I 000$)$ entre les périodes $\mathrm{I}$ et 6 a été régulière chez la vache $3 \mathrm{I} 65$; chez la vache $55 \mathrm{I} 6$, la quantité de lait produite a diminué $(2,9 \mathrm{~kg}$ en 4 semaines) avec les régimes $\mathrm{C}_{40}$ et $\mathrm{C}_{50}$, alors que le taux butyreux a augmenté (de $\mathrm{r}, 2 \mathrm{~g}$ p. I 000); puis elle a diminué lentement (de $0,6 \mathrm{~kg}$ en 6 semaines) et le taux butyreux, fortement 


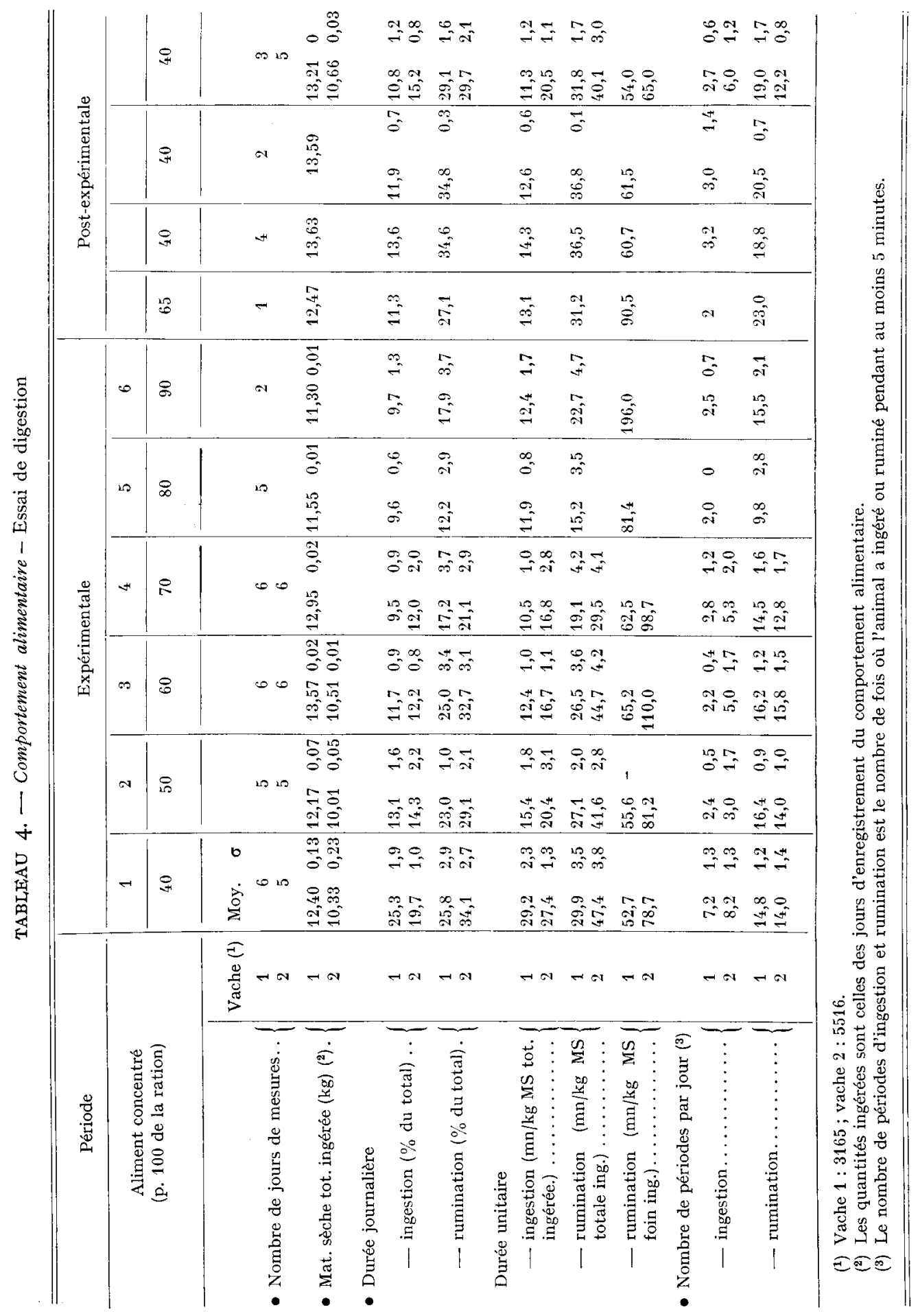


( $7 \mathrm{~g} \mathrm{p}$. I ooo). Chez les 2 animaux, la teneur du lait en matières azotées a augmenté au cours de l'essai (de $5,8 \mathrm{~g} \mathrm{p}$. I ooo et $3,0 \mathrm{~g}$ p. I ooo chez les vaches 3165 et $55 \mathrm{I} 6$ respectivement) ; le taux butyreux hebdomadaire le plus faible a été de $3 \mathrm{I}, 7 \mathrm{~g} \mathrm{p}$. I 000 .

pH du jus de rumen -.. Concentration et composition des $A G V$.

Le $\mathrm{pH}$ du jus de rumen n'a jamais été inférieur à 6,04 quelles que soient la période, la vache et l'heure du prélèvement considérés. $\mathrm{Le} \mathrm{pH}$ a toujours été maximal avant la distribution des aliments et minimal 3 ou 7 heures après (tabl. 5). La moyenne des valeurs des 4 prélèvements journaliers a été relativement élevée et n'a pas évolué de façon définie selon le régime.

\section{TABLEAU 5}

$p H$ du jus de rumen

Essai de digestion

\begin{tabular}{|c|c|c|c|c|c|c|c|c|}
\hline \multirow{2}{*}{\multicolumn{2}{|c|}{ Période }} & \multicolumn{6}{|c|}{ Expérimentale } & \multirow{3}{*}{$\begin{array}{c}\begin{array}{c}\text { Post-expéri- } \\
\text { mentale }\end{array} \\
40\end{array}$} \\
\hline & & 1 & 2 & 3 & 4 & 5 & 6 & \\
\hline \multicolumn{2}{|l|}{$\begin{array}{l}\text { Aliment concentré } \\
\text { (p. } 100 \text { de ration) }\end{array}$} & 40 & 50 & 60 & 70 & 80 & 90 & \\
\hline Heure du prélèvement $*$ & Vache $* *$ & & & & & & & \\
\hline $0 \mathrm{~h}$ & $\begin{array}{l}1 \\
2\end{array}$ & $\begin{array}{l}6,77 \\
6,54\end{array}$ & $\begin{array}{l}7,00 \\
7,25\end{array}$ & $\begin{array}{l}6,92 \\
6,72\end{array}$ & $\begin{array}{l}6,99 \\
6,98\end{array}$ & $\begin{array}{c}6,91 \\
-\end{array}$ & $\begin{array}{l}7,13 \\
-\end{array}$ & $\begin{array}{l}6,99 \\
6,94\end{array}$ \\
\hline $3 \mathrm{~h}$ & $\begin{array}{l}1 \\
2\end{array}$ & $\begin{array}{l}6,26 \\
6,14\end{array}$ & $\begin{array}{l}6,67 \\
6,50\end{array}$ & $\begin{array}{l}6,07 \\
6,41\end{array}$ & $\begin{array}{l}6,28 \\
6,45\end{array}$ & $\begin{array}{l}6,32 \\
-\end{array}$ & $\begin{array}{c}6,04 \\
-\end{array}$ & $\begin{array}{l}6,77 \\
6,60\end{array}$ \\
\hline $7 \mathrm{~h}$ & $\begin{array}{l}1 \\
2\end{array}$ & $\begin{array}{l}6,19 \\
6,17\end{array}$ & $\begin{array}{l}6,55 \\
6,77\end{array}$ & $\begin{array}{l}6,07 \\
6,20\end{array}$ & $\begin{array}{l}6,13 \\
6,31\end{array}$ & 6,10 & $\begin{array}{l}6,14 \\
-\end{array}$ & $\begin{array}{l}6,72 \\
6,73\end{array}$ \\
\hline $10 \mathrm{~h}$ & $\begin{array}{l}1 \\
2\end{array}$ & $\begin{array}{l}6,26 \\
6,40\end{array}$ & $\begin{array}{l}6,91 \\
6,87\end{array}$ & $\begin{array}{l}6,52 \\
6,66\end{array}$ & $\begin{array}{l}6,78 \\
6,75\end{array}$ & $\begin{array}{c}6,63 \\
-\end{array}$ & $\begin{array}{l}6,92 \\
-\end{array}$ & $\begin{array}{l}6,84 \\
6,81\end{array}$ \\
\hline Moyenne & $\begin{array}{l}1 \\
2\end{array}$ & $\begin{array}{l}6,37 \\
6,31\end{array}$ & $\begin{array}{l}6,78 \\
6,85\end{array}$ & $\begin{array}{l}6,40 \\
6,50\end{array}$ & $\begin{array}{l}6,55 \\
6,63\end{array}$ & 6,49 & $\begin{array}{c}6,53 \\
-\end{array}$ & $\begin{array}{c}6,83 \\
6,77\end{array}$ \\
\hline
\end{tabular}

* Par rapport à l'heure de distribution des aliments.

** Vache $1=3165$ - vache $2=5516$.

Chaque valeur est la moyenne de 6 données : 3 jours de mesure et 2 mesures pour chaque heure de prélevement (parties ventrale et dorsale du rumen).

La teneur du jus de rumen en AGV totaux a été plutôt faible (tabl. 6), surtout avant la distribution d'aliments, ce qui explique que le $\mathrm{pH}$ ait été voisin de la neutralité. Flle a été systématiquement plus élevée 3 heures après la distribution des aliments qu'avant.

La proportion de l'acide acétique a diminué quand la proportion de l'aliment concentré dans la ration a augmenté, et cette diminution a été compensée par une augmentation de la proportion de l'acide propionique et aussi, semble-t-il de l'acide 


\section{TABLEAU 6}

Concentration et composition du mélange des acides gras volatils dans le jus de rumen

Essai de digestion

\begin{tabular}{|c|c|c|c|c|c|c|c|c|c|c|c|c|c|c|}
\hline & & & & & & & & & & Post-e & expérir & imentale & & \\
\hline Période & & & & spérime & entale & & & & & & Jour ( & & & \\
\hline & & & & & & & & 1 & 2 & 3 & 5 & 6 & & \begin{tabular}{|l}
16 \\
\end{tabular} \\
\hline $\begin{array}{l}\text { Aliment conc } \\
\text { (p. } 100 \text { de la }\end{array}$ & $\begin{array}{l}\text { ntré } \\
\text { tion) }\end{array}$ & 40 & 50 & 60 & 70 & 80 & 90 & 65 & 40 & 40 & 40 & 40 & 40 & 40 \\
\hline 0 heure $\left({ }^{3}\right)$ & Vache $\left({ }^{2}\right)$ & & & & & & & & & & & & & \\
\hline $\begin{array}{l}\text { Concentration } \\
\text { (mmoles/litre) }\end{array}$ & $\begin{array}{l}1 \\
2\end{array}$ & $\begin{array}{l}86,4 \\
92,0\end{array}$ & $\begin{array}{l}80,9 \\
77,2\end{array}$ & $\begin{array}{l}78,1 \\
93,2\end{array}$ & $\begin{array}{l}87,3 \\
57,9\end{array}$ & 76,7 & 63,9 & 64,3 & 68,5 & 70,5 & 62,8 & 86,0 & 85,0 & $\begin{array}{l}67,8 \\
72,4\end{array}$ \\
\hline $\begin{array}{l}\text { Composition } \\
\text { (moles p. 100) }\end{array}$ & & & & & & & & & & & & & & \\
\hline $\mathrm{C}_{2}$ & $\begin{array}{l}1 \\
2\end{array}$ & $\begin{array}{l}69,0 \\
66,7\end{array}$ & $\begin{array}{l}67,8 \\
68,3\end{array}$ & $\begin{array}{l}67,8 \\
61,3\end{array}$ & $\begin{array}{l}65,5 \\
61,2\end{array}$ & 62,9 & 54,1 & $63, \mathbf{1}$ & 63,4 & 66,9 & 69,3 & 68,5 & 70,0 & $\begin{array}{l}69,8 \\
68,8\end{array}$ \\
\hline $\mathrm{C}_{3}$ & $\begin{array}{l}1 \\
2\end{array}$ & $\begin{array}{l}15,5 \\
16,1\end{array}$ & $\begin{array}{l}15,1 \\
15,6\end{array}$ & $\begin{array}{l}19,5 \\
20,2\end{array}$ & $\begin{array}{l}23,3 \\
19,6\end{array}$ & 16,4 & 29,1 & 18,8 & 21,2 & 20,2 & 18,4 & 17,2 & 15,9 & $\begin{array}{l}16,4 \\
17,0\end{array}$ \\
\hline Iso $\mathrm{C}_{4}$ & $\begin{array}{l}1 \\
2\end{array}$ & $\begin{array}{l}1,7 \\
1,4\end{array}$ & $\begin{array}{l}1,6 \\
1,4\end{array}$ & $\begin{array}{l}1,3 \\
0,9\end{array}$ & $\begin{array}{l}1,2 \\
0,9\end{array}$ & $1, \mathbf{4}$ & 1,7 & 1,4 & 1,4 & 0,9 & 0,9 & 1,2 & 0,9 & $\begin{array}{l}1,3 \\
0,7\end{array}$ \\
\hline $\mathrm{C}_{4}$ & $\begin{array}{l}1 \\
2\end{array}$ & $\begin{array}{l}10,5 \\
13,5\end{array}$ & $\begin{array}{l}11,5 \\
12,9\end{array}$ & $\begin{array}{r}8,6 \\
13,0\end{array}$ & $\begin{array}{r}7,7 \\
15,0\end{array}$ & 15,3 & 10,4 & 13,9 & 10,4 & 9,6 & 8,9 & 10,0 & 10,2 & $\begin{array}{r}9,9 \\
11,8\end{array}$ \\
\hline Iso $\mathrm{C}_{5}$ & $\begin{array}{l}1 \\
2\end{array}$ & $\begin{array}{l}2,4 \\
1,4\end{array}$ & $\begin{array}{l}2,1 \\
1,3\end{array}$ & $\begin{array}{l}1,8 \\
2,7\end{array}$ & $\begin{array}{l}2,2 \\
1,8\end{array}$ & 2,6 & 2,6 & 2,2 & 2,7 & 1,7 & 1,9 & 2,4 & 1,9 & $\begin{array}{l}1,5 \\
1,1\end{array}$ \\
\hline $\mathrm{C}_{5}$ & $\begin{array}{l}1 \\
2\end{array}$ & $\begin{array}{l}0,5 \\
1,0\end{array}$ & $\begin{array}{l}1,8 \\
0,5\end{array}$ & $\begin{array}{l}0,9 \\
1,7 \\
\end{array}$ & $\begin{array}{r}1,0 \\
1,4 \\
\end{array}$ & 1,4 & 2,1 & 0,5 & 0,8 & 0,6 & 0,4 & 0,7 & 0,9 & $\begin{array}{l}1,0 \\
0,5\end{array}$ \\
\hline $\begin{array}{c}3 \text { heures }\left({ }^{3}\right) \\
\text { Concentration } \\
\text { (mmoles/litre) }\end{array}$ & $\begin{array}{l}1 \\
2\end{array}$ & $\begin{array}{r}83,9 \\
110,3\end{array}$ & $\left|\begin{array}{l}95,9 \\
98,1\end{array}\right|$ & $\left|\begin{array}{r}88,7 \\
10 \%, 5\end{array}\right|$ & $\mid \begin{array}{l}98,0 \\
96,0\end{array}$ & 80,8 & 71,7 & 89,0 & 93,5 & 92,8 & 83,8 & 100,8 & 101,7 & $\begin{array}{l}88,7 \\
92,1\end{array}$ \\
\hline $\begin{array}{l}\text { Composition } \\
\text { (moles p. } 100 \text { ) }\end{array}$ & & & & & & & & & & & & & & \\
\hline $\mathrm{C}_{2}$ & $\begin{array}{l}1 \\
2\end{array}$ & $\begin{array}{l}67,5 \\
64,0\end{array}$ & $\begin{array}{l}67,8 \\
63,3\end{array}$ & \begin{tabular}{|r|}
65,3 \\
59,2 \\
\end{tabular} & $\begin{array}{l}61,0 \\
57,6\end{array}$ & 61,0 & 54,3 & 57,0 & 59,3 & 62,3 & 67,3 & 66,9 & 68,5 & $\begin{array}{l}66,1 \\
62,4\end{array}$ \\
\hline $\mathrm{C}_{3}$ & $\begin{array}{l}1 \\
2\end{array}$ & $\begin{array}{l}17,0 \\
19,6\end{array}$ & $\begin{array}{l}18,1 \\
20,3\end{array}$ & \begin{tabular}{|l|}
21,2 \\
22,3
\end{tabular} & $\begin{array}{l}24,8 \\
22,3\end{array}$ & 19,3 & 30,3 & 26,8 & 26,8 & 23,8 & 19,1 & 17,8 & 16,9 & $\begin{array}{l}19,4 \\
22,6\end{array}$ \\
\hline Iso $\mathrm{C}_{4}$ & $\begin{array}{l}1 \\
2\end{array}$ & $\begin{array}{l}1,9 \\
1,1\end{array}$ & $\begin{array}{l}1,0 \\
0,8\end{array}$ & $\begin{array}{l}1,1 \\
0,5\end{array}$ & $\begin{array}{l}0,9 \\
0,7\end{array}$ & 1,1 . & 1,2 & 0,5 & 0,5 & 0,5 & 0,5 & 0,9 & 0,8 & $\begin{array}{l}1,0 \\
0,4\end{array}$ \\
\hline $\mathrm{C}_{4}$ & $\begin{array}{l}1 \\
2\end{array}$ & $\begin{array}{r}9,7 \\
12,3\end{array}$ & $\begin{array}{l}11,3 \\
12,9\end{array}$ & $\begin{array}{r}9,7 \\
13,0 \\
\end{array}$ & $\begin{array}{l}10,6 \\
16,5\end{array}$ & 14,4 & 10,6 & 13,2 & 11,0 & 10,9 & 10,8 & 11,1 & 10,9 & $\begin{array}{l}10,8 \\
13,1\end{array}$ \\
\hline Iso $\mathrm{C}_{5}$ & $\begin{array}{l}1 \\
2\end{array}$ & $\begin{array}{l}2,3 \\
1,4\end{array}$ & $\begin{array}{l}1,4 \\
0,9\end{array}$ & $\begin{array}{l}1,6 \\
2,7\end{array}$ & $\begin{array}{l}1,5 \\
1,1\end{array}$ & 2,4 & 1,9 & 1,1 & 0,9 & 1,0 & 1,1 & 1,7 & 1,6 & $\begin{array}{l}1,3 \\
0,7\end{array}$ \\
\hline $\mathrm{C}_{5}$ & $\begin{array}{l}1 \\
2\end{array}$ & $\begin{array}{l}1,5 \\
1,5\end{array}$ & $\begin{array}{l}0,3 \\
1,8\end{array}$ & $\begin{array}{l}1,1 \\
2,2\end{array}$ & $\begin{array}{l}1,2 \\
1,7\end{array}$ & 1,6 & 1,7 & 1,4 & 1,5 & 1,4 & 1,0 & 1,6 & 1,1 & $\begin{array}{l}1,1 \\
0,7\end{array}$ \\
\hline
\end{tabular}

(1) Nombre de jours après le début du changement de régime.

(2) Vache 1:3165 - Vache 2:5516.

(3) Heure par rapport à la distribution des aliments. 
butyrique (vache $55 \mathrm{I} 6$; vache $3 \mathrm{I} 65$ avec le régime $\mathrm{C} 80$ ). Cependant, avec le régime C 9o, la proportion de l'acide butyrique a diminué. D'une façon systématique, la proportion de l'acide acétique a été plus faible avant la distribution d'aliments qu'après, et la proportion de l'acide propionique a été plus élevée; celle de l'acide butyrique n'a pas varié.

Pendant la semaine de transition pour la vache $3 \mathrm{I} 65$, la composition du mélange des $\mathrm{AGV}$ est redevenue semblable à celle observée avec le régime $\mathrm{C} 50$ dès le $4{ }^{-} 5^{\mathrm{e}}$ jour après le début du changement de régime alimentaire. Ce résultat est en accord avec ce qu'ont observé STORRy et SutTon (I969). Pour la vache 55I6, elle n'a été mesurée que 2 semaines après le changement de régime; elle était alors semblable à celle observée avec le régime $\mathrm{C} 50$.

La somme des proportions des $\mathrm{AGV}$ précurseurs des acides gras du lait $\left(\mathrm{C}_{2}+\mathrm{C}_{4}\right)$ a peu diminué jusqu'au régime $\mathrm{C} 80$ chez la vache 3 I 65 et $\mathrm{C} 70$ chez la vache $55 \mathrm{I} 6$. En revanche, le rapport $\mathrm{C}_{2} / \mathrm{C}_{3}$ a rapidement diminué chez les 2 vaches.

\section{TABLEAU 7}

Teneur du sang en corps cétoniques et du plasma en acides gras libres et en glucose Essai de digestion

\begin{tabular}{|c|c|c|c|c|c|c|c|c|c|c|c|}
\hline \multirow{3}{*}{\multicolumn{2}{|c|}{ Période }} & \multirow{2}{*}{\multicolumn{6}{|c|}{ Expérimentale }} & \multicolumn{4}{|c|}{ Post-expérimentale } \\
\hline & & & & & & & & \multicolumn{2}{|c|}{$\mathrm{S}_{1}^{*}$} & \multirow{2}{*}{$\frac{\mathrm{S}_{2^{*}}}{40}$} & \multirow{2}{*}{$\frac{\mathrm{S}_{3}{ }^{*}}{40}$} \\
\hline & & 40 & 50 & 60 & 70 & 80 & 90 & 65 & $t_{1} 0$ & & \\
\hline \multicolumn{12}{|l|}{$\begin{array}{l}\text { Acides gras libres } \\
\text { ( } \mu \text {-éq/l de plasma) }\end{array}$} \\
\hline $0 \mathrm{~h}$ & $\begin{array}{l}1 \\
2\end{array}$ & $\begin{array}{l}496 \\
332\end{array}$ & $\begin{array}{l}329 \\
466\end{array}$ & $\begin{array}{l}349 \\
316\end{array}$ & $\begin{array}{l}162 \\
190\end{array}$ & $28^{\prime}$ & 395 & 162 & 253 & 324 & $\begin{array}{l}379 \\
248\end{array}$ \\
\hline $3 \mathrm{~h}$ & $\begin{array}{l}1 \\
2\end{array}$ & $\begin{array}{l}373 \\
292\end{array}$ & $\begin{array}{l}228 \\
248\end{array}$ & $\begin{array}{l}211 \\
192\end{array}$ & $\begin{array}{r}174 \\
98\end{array}$ & 176 & 154 & 174 & 174 & 224 & $\begin{array}{l}190 \\
144\end{array}$ \\
\hline \multicolumn{12}{|l|}{$\begin{array}{l}\text { Corps cétoniques totaux } \\
\text { (mg acétone/100 ml } \\
\text { de sang) }\end{array}$} \\
\hline $0 \mathrm{~h}$ & $\begin{array}{l}1 \\
2\end{array}$ & $\begin{array}{l}1,82 \\
1,51\end{array}$ & $\begin{array}{l}1,72 \\
1,75\end{array}$ & $\begin{array}{l}1,51 \\
1,87\end{array}$ & $\begin{array}{l}1,40 \\
1,75\end{array}$ & 1,59 & 1,52 & 1,92 & 1,71 & 1,60 & $\begin{array}{l}2,09 \\
1,70\end{array}$ \\
\hline $3 \mathrm{~h}$ & $\begin{array}{l}1 \\
2\end{array}$ & $\begin{array}{l}1,85 \\
1,96\end{array}$ & $\begin{array}{l}2,22 \\
2,05\end{array}$ & $\begin{array}{l}1,88 \\
2,50\end{array}$ & $\begin{array}{l}2,30 \\
2,98\end{array}$ & 2,48 & 2,11 & 2,20 & 1,83 & 2,35 & $\begin{array}{l}2,08 \\
2,03\end{array}$ \\
\hline $\begin{array}{c}\text { Glucose } \\
\text { (mg/litre de plasma) }\end{array}$ & & & & & & & & & & & \\
\hline $0 \mathrm{~h}$ & $\begin{array}{l}1 \\
2\end{array}$ & $\begin{array}{l}742 \\
766\end{array}$ & $\begin{array}{l}665 \\
771\end{array}$ & $\begin{array}{l}737 \\
699\end{array}$ & $\begin{array}{l}736 \\
678\end{array}$ & 716 & 739 & 564 & 742 & 664 & $\begin{array}{l}657 \\
673\end{array}$ \\
\hline $3 \mathrm{~h}$ & $\begin{array}{l}1 \\
2\end{array}$ & $\begin{array}{l}726 \\
750\end{array}$ & $\begin{array}{l}605 \\
701\end{array}$ & $\begin{array}{l}703 \\
697\end{array}$ & $\begin{array}{l}672 \\
624\end{array}$ & 662 & 697 & 582 & 718 & 680 & $\begin{array}{l}662 \\
686\end{array}$ \\
\hline
\end{tabular}

* $\mathrm{S}_{1}, \mathrm{~S}_{2}, \mathrm{~S}_{3}:$ Semaine 1, 2, 3 .

** Vache 1 : 3165 - Vache 2 : 5516. 
Pour une période expérimentale et une vache données, la variation d'un jour à l'autre de la proportion des principaux AGV a été faible même quand la proportion d'aliment concentré dans l'aliment était élevée.

Le taux butyreux a été assez étroitement lié à la composition du mélange des $\mathrm{AGV}$ du jus de rumen; les coefficients de corrélation ont été de $+0,83 ;-0,64$ et $+0,67$ avec les proportions molaires de $\mathrm{C}_{2}, \mathrm{C}_{3}$ et $\mathrm{C}_{2}+\mathrm{C}_{4}$ respectivement pour la vache $3 \mathrm{I} 65$, et de $+0,83 ;-0,97$ et $+0,88$ pour la vache $55 \mathrm{I} 6$.

Teneur du sang en certains constituants.

Les teneurs du sang ou du plasma en acides gras libres, en corps cétoniques totaux et en glucose ont été semblables chez les 2 animaux et n'ont pas évolué dans un sens défini d'un régime à l'autre, sauf la teneur du plasma en acides gras libres 3 heures après le repas qui a diminué d'une période expérimentale à la suivante (tab1. 7). La teneur en acides gras libres et glucose a été plus élevée avant la distribution des aliments que 3 heures après (de respectivement 55 et 3,7 p. IOO), mais celle en corps cétoniques totaux a été plus faible (de 23 p. roo).

Comparée à la composition du sang des vaches normales, celle du sang des vaches fistulées se distingue par une teneur plus élevée en glucose et plus faible en corps cétoniques.

\section{DISCUSSION}

Dans l'essai d'alimentation précédent (RÉmond et JouRnE'T, I97I), les vaches recevaient l'aliment concentré et le fourrage à volonté et ingéraient environ $85 \mathrm{p}$. Ioo d'aliment concentré; le taux butyreux avait été faible (en moyenne $23 \mathrm{~g} \mathrm{p}$. I ooo) et la quantité de lait produite avait diminué d'une façon anormalement rapide.

Les résultats de l'essai d'alimentation que nous venons de rapporter différent de ceux de l'essai précédent par essentiellement 2 faits : $\mathbf{I}^{\circ} /$ pour une même proportion d'aliment concentré dans la ration, le taux butyreux a été plus élevé; $2^{\circ} / 1 a$ quantité de lait produite à $4 \mathrm{p}$. Ioo de matières grasses a diminué à peu près de la même façon que la quantité d'énergie ingérée par les animaux. Comment expliquer ces différences?

Dans cet essai les quantités d'aliments distribuées étaient limitées et faibles; les animaux n'étaient pas rassasiés : c'est ce qu'indique le groupement des périodes d'ingestion après la distribution des aliments, et la faible durée unitaire d'ingestion.

La limitation des quantités ingérées explique probablement en grande partie que la composition du mélange des AGV semble avoir moins différé d'une composition normale que dans l'essai précédent (si on excepte le régime $\mathrm{C} 90$ ) ; ces résultats confirmeraient ceux de BATH et RooK (I963) qui ont montré que l'augmentation du niveau d'alimentation diminuait la proportion de 1'acide acétique, surtout quand les régimes étaient riches en aliment concentré. La présentation broyée de l'aliment concentré (au lieu de agglomérée dans l'essai précédent) a probablement aussi contribué au maintien d'une composition normale du mélange des AGV.

En raison des modifications limitées des fermentations dans le rumen, le taux butyreux n'a que modérément diminué; de plus, à cause du rationnement de 1'énergie offerte, les quantités absorbées d'acide propionique et peut-être aussi de glucose n'ont pas dû être assez importantes pour modifier le métabolisme lipidique de l'ani- 
mal et provoquer un engraissement important. Le bilan énergétique positif et peu variable d'un régime à l'autre, l'efficacité constante de l'utilisation énergétique des régimes et la teneut constante du plasma en AGI, et en glucose viennent à l'appui de l'hypothèse selon laquelle la part de l'énergie utilisée pour la synthèse du lait a peu varié.

L'efficacité à peu près constante d'utilisation de l'énergie nette quelle que soit la proportion d'aliment concentré confirme la majorité des résultats déja obtenus (cf. introduction). Cependant dans notre essai, la diminution trop rapide de la quantité d'énergie distribuée aux animaux a peut-être empêché la quantité d'énergie produite dans le lait de diminuer plus rapidement qu'elle. On peut done se demander si nous aurions obtenu les mêmes résultats en diminuant moins rapidement la quantité d'énergie offerte aux animaux. La faible efficacité alimentaire des différents régimes que nous avons malgré tout observée est-elle due au fait que les animaux ont utilisé une partie de l'énergie ingérée pour leurs synthèses corporelles? L'augmentation du poids vif entre la période I et de la dernière semaine de la période postexpérimentale, et la teneur relativement faible du plasma en AGL suggèrent que le poids vif vide des animaux a effectivement augmenté et que le bilan énergétique était positif. Cependant, de la façon dont nous l'avons calculée, la valeur du bilan énergétique est entachée d'incertitude provenant de l'évaluation : a) des besoins des animaux; nous avons adopté les recommandations des besoins d'entretien de vaches adultes alors que la plupart étaient encore en croissance ( 8 étaient en première lactation), b) des quantités d'énergie ingérées par les animaux. La valeur énergétique des aliments a seulement été estimée (voir paragraphe Dispositif Expérimental) et a pu varier avec le régime à cause de la diminution de digestibilité de la cellulose brute dans les rations riches en aliment concentré.

A condition que les quantités distribuées aux animaux soient limitées, il semble possible d'alimenter des vaches laitières avec des régimes riches en aliment concentré sans que cela entraîne une modification, du moins importante, de l'utilisation de l'énergie ingérée et bien que le lait ait un faible taux butyreux. La portée de nos résultats est cependant limitée par la courte durée de l'essai que nous avons fait et le niveau de production laitière moyen des animaux; il serait nécessaire de les confirmer avec des vaches de niveau de production élevé et sur des périodes plus longues. Il est d'ailleurs possible que ces vaches puissent être alimentées presque à volonté puisque GEAY (communication personnelle) a montré avec des taurillons en croissance qu'une faible diminution des quantités par rapport au niveau ad libitum améliorait l'utilisation énergétique de la ration.

Rçu pour publication en juin 1972.

\author{
SUMMARY
}

\title{
FEEDING OF DAIRY COWS WITH RATIONS OF VARIED CONCENTRATE LEVELS : MILK PRODUCTION AND COMPOSITION, AND DIGESTION IN THE RUMEN
}

During 5 or 6 successive experimental periods ten normal cows and two fitted with a large rumen fistula, all lactating, received previously determined amounts of feed with concentrate levels from 50 or 40 to 90 p. IOO (IO p. Ioo increase at each period). After the last experimental, 
period, the concentrate level of the rations was reduced again, in two days, to 50 p. Ioo (post experimental period).

The animals always consumed nearly the whole ration offered in the two hours following the feeding (tables 2 and 4 ). The 4 p. Ioo milk yield decreased a little more rapidly than the milk equivalent of the net energy supply $(0.48 \mathrm{~kg}$ versus $0.44 \mathrm{~kg}$ per week, fig. I). The fat content of milk decreased in a linear way ( $\mathrm{r} .5 \mathrm{~g}$ p. I 000 per period), but it became almost normal again in the course of the second post-experimental week. The live weight variation was similar to that of the fat content. The energy balance of the animals was always positive (on average I. I FU) during the different experimental periods and the blood plasma content of free fatty acids was rather low (table 3). The proportion of acetic acid in the mixture of volatile fatty acids decreased when the concentrate level of the diet became higher, whereas that of propionic acid increased; these modifications were, however, relatively limited (table 6).

The changes as regards digestion in the rumen as well as production and composition of milk were much smaller than those observed in the course of the two previous trials with ad libitum feeding of concentrates (RÉMOND and JOURNET, I97 I and 1972).

\section{RÉFÉRENCES BIBLIOGRAPHIQUES}

BAтн I. H., Rook J.A. F., I963. The evaluation of cattle foods and diets in terms of the ruminal concentration of volatile fatty acids. I. The effects of level of intake, frequency of feeding, the ratio of hay to concentrates with diet, and of supplementary feeds. J. Agric. Sci., 61, 34I-348.

Bell J. W., Horton O. H., Stallcup O. T., ig63. Effect of high versus normal concentrate roughage ration on digestibility, milk production and efficiency of production. J. Dairy Sci, 46, 623 (Abstr.).

Bishop S. E., Loosli J. K., Trimberger G. W., TURK K. L., I963. Effects of pelleting and varying grain intakes on milk yield and composition. J. Dairy Sci., 46, 22-26.

Bloom S., Jacobson N. L., Mc Gilliard L. D., Heady E. O., Homeyer P. G., I955. Effects of various hay : concentrate ratios on production responses and nutrient utilization of dairy cows. $J$. Dairy Sci., 38, 6r4 (Abstr.).

Breirem K., 1954. Die nettoenergie als grundlage der Bewertung der Futtermittel, in : Nehring K., Ioo Jahre möckern. Die Bewertung der Futterstoffe tınd andere. Probleme der Tiernährung. Berlin, Deutsche Akad. der Landwirtschaftswissenschaften, t. 2, 97-I08.

Brown L. D., Thomas J. W., Emery R. S., Mc Gilliard L. D., Armstrong O. V., Lassiter C. A., I962. Effect of high-level grain feeding on milk production response of lactating dairy cows. J. Dairy Sci., 45, I $184-1187$.

Coppock C. E., Flat W. P., Moore L. A., Stewart W. E., Ig64. Effect of hay to grain ratio on utilization of metabolizable energy for milk production by dairy cows. J. Dairy Sci., 47, I330-1338.

Dole V. P., Meinertz H., I96o. Determination of long-chain fatty acids in plasma and tissues. $J$. Biol. Chem., 235, 2595-2599.

Elliott J. M., Moosli J. K., I959. Effect of the dietary ratio of hay to concentrate on milk production, ration digestibility and urinary energy losses. J. Dairy Sci., 42, 836-842.

Etgen W. M., Bhatt B. M., Henderson Jr. B. W., Berolsek E. R., Gilbert R. W., i965. Effect of forage-concentrate ratios on lactating dairy cows. J. Dairy Sci., 48, I5 8 (Abstr.).

Hinders R. G., OWen F. G, I963. Relationships between efficiency of milk production and ruminal volatile fatty acids of cows fed isocaloric (ENE) rations of varied concentrate levels. J. Dairy Sci., 46, $1246-1250$.

Hooven N. H., Plowman R. D., 1963. Ad libitum feeding of dairy cattle. J. Dairy Sci., 46, 622 (abstr.).

Huggett A. S. G., Nixon D. A., 1957. Enzymic determination of blood glucose. Biochem. J., 66, I $2 \mathrm{p}$.

Martin T. G., Stondard G. E., Alinn R. S., i954. The effects of varied rates of hay feeding on body weight and production of lactating dairy cows. J. Dairy Sci., 37, I233-1240.

Morris J. L., Gainer F., Davis R. F., I958. Studies on forage : concentrate ratios for milk production. J. Dairy Sci., 41, $72 \mathrm{I}$ (Abstr.).

Nordfeldt S., RUUdvere A., I963. Influence of various levels of roughage and concentrate and the effects of different planes of nutrition on milk production in dairy cows. Lantbrukshsökolans Annaler, 29, $345-393$.

Olson H. H., Hinners S. W., Bernett R. C., I966. Ad libitum versus restricted concentrate feeding of lactating dairy cows. J. Dairy Sci., 49, IIO-II3.

Procos J., I96I. Modification of the spectrophotometric determination of ketone bodies on blood enabling the total recovery of $\beta$-hydroxybutyric acid. Clin. Chem., $7,97-106$. 
Putnam P. A., Loosli J. K., 1959. Effect of feeding different ratios of roughage to concentrate upon milk production and digestibility of the ration. J. Dairy Sci., 42, ro70-1078.

Rémond B., Journet M., I97r. Alimentation des vaches laitières avec des rations à forte proportion d'aliments concentrés. I. Quantités ingérées et production laitière. Ann. Zootech., 20, I69-184.

RÉmond B., Journet M., I972. Alimentation des vaches laitières avec des rations à forte proportion d'aliments concentrés. II. Comportement alimentaire et digestion dans le rumen. Ann. Zootech. 21, I9I-205

Rigaud J., Journet M., I970. Méthode de dosage des acides gras volatils dans le liquide du rumen. Ann. Biol. anim. Bioch. Biophys., 10, I5 I-I57.

RoNNing M., I960. Effect of varying alfalfa hay-concentrate ratios in a pelleted ration for dairy cows. J. Dairy Sci., 43, 8 I I-8I5.

Ruckebush Y., 1963. Recherches sur la régulation centrale du comportement alimentaire chez les ruminants. Thèse Doct. Sci., Lyon.

Storry J. E., Sutton J. D., Ig69. The effect of change from low-roughage to high-roughage diets on rumen fermentation, blood composition and milk fat secretion in the cows. Br. J. Nutr., 23, 5 II -52 I

Swanson E. W., Hinton S. A., Miles J. T., I967. Full lactation response on restricted ad libitum roughage diets with liberal concentrate feeding. J. Dairy Sci., 50, I I47-I I52.

Zeremski D., Van Horn H. H., Mc Gilliard A. D., Jacobson N. L., rg65. Effect of the net energy concentration of total ration on milk production and composition. J. Dairy Sci., 48, 1467-1472. 